\title{
Article \\ Elucidating the Mechanical Energy for Cyclization of a DNA Origami Tile
}

\author{
Ruixin Li $\mathbb{D}$, Haorong Chen, Hyeongwoon Lee and Jong Hyun Choi *๑
}

School of Mechanical Engineering, Purdue University, West Lafayette, IN 47907, USA; li2594@purdue.edu (R.L.); haorongc@mit.edu (H.C.); lee3807@purdue.edu (H.L.)

* Correspondence: jchoi@purdue.edu

Citation: Li, R.; Chen, H.; Lee, H.; Choi, J.H. Elucidating the Mechanical Energy for Cyclization of a DNA Origami Tile. Appl. Sci. 2021, 11, 2357. https://doi.org/10.3390/ app11052357

Academic Editor: Alexander E. Marras

Received: 7 February 2021

Accepted: 3 March 2021

Published: 6 March 2021

Publisher's Note: MDPI stays neutral with regard to jurisdictional claims in published maps and institutional affiliations.

Copyright: (c) 2021 by the authors. Licensee MDPI, Basel, Switzerland. This article is an open access article distributed under the terms and conditions of the Creative Commons Attribution (CC BY) license (https:// creativecommons.org/licenses/by/ $4.0 /)$.

\begin{abstract}
DNA origami has emerged as a versatile method to synthesize nanostructures with high precision. This bottom-up self-assembly approach can produce not only complex static architectures, but also dynamic reconfigurable structures with tunable properties. While DNA origami has been explored increasingly for diverse applications, such as biomedical and biophysical tools, related mechanics are also under active investigation. Here we studied the structural properties of DNA origami and investigated the energy needed to deform the DNA structures. We used a single-layer rectangular DNA origami tile as a model system and studied its cyclization process. This origami tile was designed with an inherent twist by placing crossovers every 16 base-pairs (bp), corresponding to a helical pitch of $10.67 \mathrm{bp} /$ turn, which is slightly different from that of native B-form DNA ( 10.5 bp/turn). We used molecular dynamics (MD) simulations based on a coarse-grained model on an open-source computational platform, oxDNA. We calculated the energies needed to overcome the initial curvature and induce mechanical deformation by applying linear spring forces. We found that the initial curvature may be overcome gradually during cyclization and a total of $\sim 33.1 \mathrm{kcal} / \mathrm{mol}$ is required to complete the deformation. These results provide insights into the DNA origami mechanics and should be useful for diverse applications such as adaptive reconfiguration and energy absorption.
\end{abstract}

Keywords: DNA nanotechnology; DNA origami; self-assembly; DNA helicity; mechanics; deformation; cyclization; MD simulations; numerical analysis; oxDNA

\section{Introduction}

DNA self-assembly is a powerful bottom-up manufacturing approach based on spontaneous sequence complementarity [1,2]. DNA nanotechnology can produce complex architectures with sub-nanometer accuracy and precision, using DNA tiles [3-5] and DNA bricks [6-8]. The tile-based assembly uses several short single-stranded DNA (ssDNA) oligonucleotides to assemble one or few motifs, which then form two-dimensional (2D) or three-dimensional (3D) configurations by using their sticky ends. In contrast, each DNA brick is a unique ssDNA strand, thus more intricate geometries may be constructed from a single step annealing reaction. Dynamic DNA nanostructures are also available, including tweezers [9,10] and walkers [11-14]. These mobile nanodevices rely on toehold-mediate strand displacement reaction [15-17], to detach from some of the strands in order to stay undefined or bind with new strands. The major difference between the two devices is the designed movement; DNA tweezers mostly switch between open and closed states, while DNA walkers can move along a track as long as fuel strands are available.

DNA origami is a widely used method due to excellent programmability and structural predictability [18-23]. In this approach, a long ssDNA 'scaffold' strand (up to several thousand base-pairs or bp) is laid out in a folded pattern to approximate the target design. A number of short ssDNA 'staple' strands are then added to bring selected parts of scaffold together by hybridization to form designed conformations. Various 2D and 3D DNA origami structures have been demonstrated, including shafts [24-26], tiles [27-30], cubes [31-33], 
and wireframe polygons [34-36], to name a few. Dynamic, shape-changing DNA origami structures were also realized via two-step reactions: strand displacement and reannealing [37-39]. While the strand displacement reaction is possible at room-temperature due to the low energy barrier, the reannealing with new strands is often performed at elevated temperatures to provide necessary activation energies [40-42]. For both static and dynamic DNA origami architectures, their structural properties and mechanical behaviors are of great interest, including the twisting of a double-stranded DNA (dsDNA) bundle [43,44], the interconnection between bundles by crossovers [45], the interaction between ds-regions in an origami structure [46], and the structural deformation by applied external forces [47]. A better understanding of such fundamental mechanics will significantly advance the design and development of DNA origami for a variety of applications.

There are two typical lattices (honeycomb and square) for cross-section designs of DNA origami. These two design approaches may be distinguished by crossover arrangements as they are distinct and different based on the layouts of dsDNA bundles and crossovers. In a honeycomb lattice, a crossover is placed every $2 / 3$ turn. Given the helicity of around 10.5 $\mathrm{bp} /$ turn for native B-form DNA, it is exactly 7 bp per crossover [48-50]. In contrast, a crossover is placed every $3 / 2$ turns in a square lattice, yielding $15.75 \mathrm{bp}$ per crossover. However, only an integer number of base pairs is possible in the design. Thus, $16 \mathrm{bp}$ per crossover is used frequently, which corresponds to $10.67 \mathrm{bp} /$ turn. The difference between 10.67 and 10.5 $\mathrm{bp} /$ turn in DNA helicity causes a right-handed twist in the entire structure $[20,24,27]$. The structural twist and curvature may be resolved by forcing the upper and lower boundaries together using linker staples [27] or by adding intercalators that directly modulate the helicity (e.g., from 10.5 to $10.67 \mathrm{bp} /$ turn) [24,51-53]. Regardless of the methods, external (mechanical or chemical) energy will be accounted for the structural correction, and this is the activation energy required for the deformation of the origami.

In this work, we investigated a cyclization process of a single-layer DNA origami rectangle. This origami tile is designed in a square lattice and thus has an inherent structural curvature. In a previous publication [27], we used this tile in a series of experiments to study the energy needed for cyclization as functions of incubation temperature, origami size, and structural defect. The atomic force microscopy (AFM) measurements and mechanical analysis of elastic deformation were performed to provide insights into the reconfiguration, including relevant reaction rates and the bending spring constant of crossovers. While the experiment and elasticity theory revealed the overall aspects of the origami deformation, the detailed process was not known, because only the DNA conformations before and after the cyclization reaction were available. In the current study, we performed numerical simulations of the DNA origami tile cyclization by directly applying external forces. The multistep simulations of small, quasi-equilibrium deformations allowed us to elucidate the details of the entire cyclization process and calculate the mechanical energy associated with conformational change at each step. The computation was performed with coarse-grained molecular dynamics (MD) model in oxDNA, an open-source computational platform. The MD computation was also complemented by finite element method (FEM) simulations in CanDo, a free, online simulation software. The evolution of the simulated structure in each state shows how the DNA origami tile transforms gradually from its initial twisted conformation to the final cyclized configuration. The overall energy for the complete cyclization can be calculated by summing all the mechanical energies in the multistep simulations and is approximately $33.1 \mathrm{kcal} / \mathrm{mol}$. In addition, we also calculated the energy needed to flatten the twisted DNA origami tile, which is $\sim 26.7 \mathrm{kcal} / \mathrm{mol}$. The energies for cyclization and flattening are in excellent agreement with previous experimental and theoretical results [27].

\section{Materials and Methods}

\subsection{Origami Tile Design}

Figure 1 shows the design of a single-layer DNA origami rectangle used as a model system in this computational study. There are 32 helices in height (i.e., 32 rows of ds- 
DNA bundles) and 224 bases in width (in each row). The origami tile is sketched in caDNAno2 [48]. To avoid blunt-ended stacking, only 192 bases in width are used and the 16 nucleotides (nt) on either side are left unpaired. The sequence information is available in our previous report [27]. The designed width is calculated to be approximately $64 \mathrm{~nm}$ based on $0.332 \mathrm{~nm} / \mathrm{bp}$ along the axis of regular, right-handed B-form DNA. The height is estimated using the diameter of a dsDNA bundle $(\sim 2.2 \mathrm{~nm})$ and the distance between the center of neighboring bundles ( 2.3 nm when closely packed) [20,46]. However, the gap between adjacent bundles is noticeably wider than the bundle diameter based on our previous AFM results [27]. For a fair estimation, we assume the gap to be approximately $2.3 \times 1.2 \mathrm{~nm}$. Thus, the designed height is estimated as $\sim 88 \mathrm{~nm}$. A set of linker staples, if introduced in an experiment, can hybridize with upper and lower boundaries (rows 1 and 32) simultaneously, thereby cyclizing the tile into a tube, as we demonstrated in a previous report [27]. Experimentally, the DNA tile experiences thermal fluctuation that deforms the structure randomly, and the linkers seal the upper and lower edges when they are in proximity. By design, the cyclized tube is approximately $64 \mathrm{~nm}$ in width and $28 \mathrm{~nm}$ in diameter. If collapsed to a flat surface, the origami will be about $64 \mathrm{~nm}$ in width, $44 \mathrm{~nm}$ in height, and $4 \mathrm{~nm}$ in thickness (double layer). The AFM results suggest that the synthesized tile measures about $90 \times 65 \times 2 \mathrm{~nm}$ and the flattened tube is around $45 \times 65 \times 4 \mathrm{~nm}$ [27].

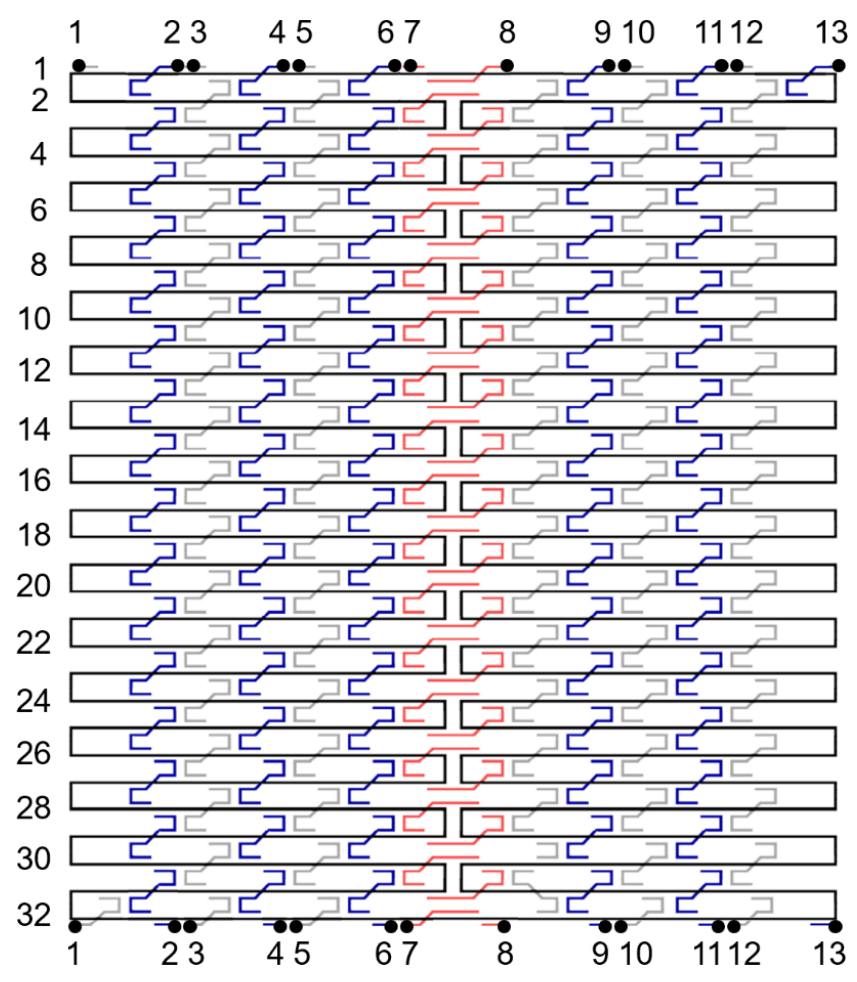

Figure 1. Folding path diagram of a DNA origami tile. Black line denotes the scaffold strand, which routes clockwise ( 5 ' to $3^{\prime}$ end). Blue, gray, and red lines represent staples. The color marks different shape and directions. Blue and gray staples have the same shape but opposite directions: blue goes down while gray goes up from 5' to 3' end. The red staples secure the scaffold in the middle of the origami and thus have a different shape compared with blue and gray. A typical staple length is $32 \mathrm{nt}$. The dsDNA bundles are numbered 1 through 32, as noted on the left. Note that a total of 13 gray and blue staples were used to seal the upper and lower edges to form a cyclized tube in the experiment. The 13 pairs of the black dots indicate the cut sites of the linker staples. This design was used in the MD simulations.

The DNA origami tile is designed in a square lattice, with a crossover every $16 \mathrm{nt}$. Typically a staple is $32 \mathrm{nt}$ in length-the middle $16 \mathrm{nt}$ binds with a dsDNA helix while the $8 \mathrm{nt}$ parts at both $5^{\prime}$ and $3^{\prime}$ ends bind with neighboring two dsDNA bundles. This is equivalent to 
a helicity of $10.67 \mathrm{bp} /$ turn. Therefore, the tile will have a slight, right-handed global twist if subjected under $10.5 \mathrm{bp} /$ turn conditions. In the simulations, 13 linker staples designed to cyclize the DNA origami rectangle were cut into halves as illustrated in Figure 1. The cutting sites are marked with 13 pairs of black dots and labelled 1 through 13. The origami tile can then be cyclized by applied external forces.

\subsection{FEM Simulations with CanDo}

FEM is a numerical method for solving partial differential equations (PDEs) in 2D or 3D space variables. The method subdivides a large system into smaller, simpler parts that are called finite elements to solve the equations. In our case, FEM simulations were performed with CanDo on single base-pair resolution to study 3D equilibrium conformations of the DNA origami tile (without linker staples). The axial rise per base-pair and the helix diameter were set at 0.34 and $2.25 \mathrm{~nm}$. The crossover spacing was $10.5 \mathrm{bp}$. All the mechanical properties of dsDNA were set as default: axial stiffness at $1100 \mathrm{pN}$, bending stiffness at $230 \mathrm{pN} \cdot \mathrm{nm}^{2}$, torsional stiffness at $460 \mathrm{pN} \cdot \mathrm{nm}^{2}$. To simplify the properties of ssDNA, nick stiffness factor was set at 0.01 , which accounts for the property ratio between ssDNA and dsDNA. For example, the axial stiffness of ssDNA is $0.01 \times 1100 \mathrm{pN}$, which is $11 \mathrm{pN}$. The simulations yield an inherent global twist in the origami conformation.

\subsection{Simulations with OxDNA}

MD computation is a method for analyzing the physical movements of atoms and molecules (particles). All-atom models include all the related atoms in the simulations for better accuracy, while coarse-grained models use a pseudo-atom to represent a group of atoms to save simulation time. The particles are subject under interactions for a fixed duration of time, giving a view of the dynamic evolution of the system. The trajectories of the particles are typically determined by numerically solving Newton's equations of motion for the particles.

The MD simulations based on a coarse-grained model were performed with oxDNA [54] to study equilibrium conformations of the DNA origami tile and their gradual structural deformations in series during cyclization and flattening by external loading. Specifically, the oxDNA2 model was used with the temperature at $300 \mathrm{~K}$. In experiments, $\mathrm{Mg}^{2+}$ is typically used to mitigate the repulsion between negatively charged DNA strands. However, there is no $\mathrm{Mg}^{2+}$ concentration in the oxDNA simulations. Instead, the salt concentration was set at $\left[\mathrm{Na}^{+}\right]=0.5 \mathrm{M}$ [54-57]. The caDNAno2 file of the origami tile was converted into topology and configuration files as the initial conformation in oxDNA with actual sequence information. To simplify the computation, all the ss-regions that do not serve any structural purposes (e.g., 8-nt toeholds on linker staples and free scaffold loops) were not included in the initial conformation. Since these regions were barely seen interfering with dsDNA bundles under AFM, it is reasonable to exclude them. As discussed in the 'Origami Tile Design' section, the linker staples were cut into two halves so that the upper and lower boundaries were separate before structural deformation. The configuration was relaxed by MD simulations without any external forces for $2 \times 10^{6}$ steps to obtain the initial equilibrium structure.

In the cyclization process, external linear spring forces were introduced by mutual traps in oxDNA (see oxDNA documents for details) and applied on the cut sites of the linkers (13 pairs of black dots located at the upper and lower boundaries in Figure 1) on origami tile to induce a cyclization through a series of deformations. Similar spring forces were used to study origami deformations in other reports $[55,58]$. The potential energy associated with the spring forces was determined by considering the conformation in each state. Figure 2a shows the loading on a pair of nucleotides. A hypothetical spring that connects nucleotide $\mathrm{A}$ and $\mathrm{B}$ has a spring force constant $k$ and an equilibrium length $l_{0}$. Given the distance of A and B is $l$, the forces can be expressed following the Hooke's law.

$$
F_{A}=F_{B}=k\left(l-l_{0}\right)
$$


(a)

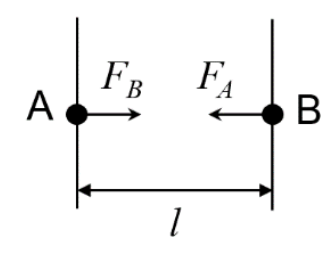

(b)

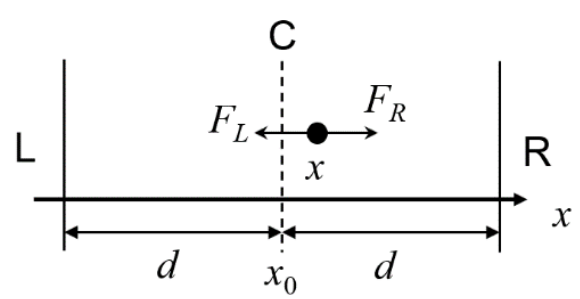

Figure 2. Mechanical loadings in oxDNA simulations. (a) Hypothetical linear spring forces between two nucleotide A and B (solid dots) with a distance $l$. The forces on A pointing to B is denoted as force $F_{B}$, and similar naming applies to $F_{A}$. A set of spring forces was used for cyclization of the DNA origami tile. (b) Hypothetical linear spring forces pulling a nucleotide (solid dot) at $x$ to an imaginary plane (dashed line, marked as $\mathrm{C}$ ) located at $x_{0}$. The forces are realized by two planes, $\mathrm{L}$ and $\mathrm{R}$ (vertical lines), in a distance $d$ from the imaginary plane on the left and right sides. The force toward the left plane (L) is $F_{L}$, whereas the force toward the right plane (R) is $F_{R}$.

Assuming the potential energy is 0 at $l=l_{0}$, the potential energy of the spring system can be calculated.

$$
E_{A B}=0.5 k\left(l-l_{0}\right)^{2}
$$

For a given conformation of the origami tile, $l$ is set. The potential energy can be modulated by varying $k$ and $l_{0}$ (i.e., changing the spring) in each calculation from a state to the next. Overall, two million calculation steps were carried out, which took about two days of computation. After each calculation, the results were analyzed by visualizing the structures in cogli2 and by extracting the directional and positional data. The conformation with the minimum of global potential energy was chosen as the next state. The distance between two nucleotides from the cut of each linker staple was measured from the positional data to obtain a vector pointing from one terminal to the other.

In the flattening process, external linear spring forces were introduced by two force planes in oxDNA (see oxDNA documents for details) and applied on all 13,360 nucleotides of the origami tile to force them to an imaginary center plane through a series of deformations. If the plane is aligned perpendicular to the $x$-axis, the loading of any single nucleotide will be as shown in Figure 2b. We placed two force planes for loading. The force toward the left plane (L) is $F_{L}$, whereas the force toward the right plane (R) is $F_{R}$. Each plane exerts a spring force constant $k$ in a distance $d$ from the center plane (dashed line, C). Given that the positions of the center plane and the nucleotide are $x_{0}$ and $x$, respectively, the forces are

$$
\begin{aligned}
& F_{L}=k\left[x-\left(x_{0}-d\right)\right] \\
& F_{R}=k\left[\left(x_{0}+d\right)-x\right]
\end{aligned}
$$

Note that the directions of the forces are marked in Figure 2b. If the positive direction is set as the direction of the $x$-axis, the total force is

$$
F=F_{R}-F_{L}=2 k\left(x_{0}-x\right)
$$

Thus, the actual direction of the total force $F$ is always pointing to the center plane and the magnitude is proportional to the distance of the nucleotide from the center plane. Note that the force is independent of $d$. Similar to the two nucleotides linked by a spring force pair as shown in Figure 2a, the potential energy of the spring system may be determined with an assumption that the potential energy is 0 at $x=x_{0}$.

$$
E=k\left(x_{0}-x\right)^{2}
$$

For a given origami conformation, $x$ is set. The location of the center plane is determined by the initial state (state 0 ), which means that $x_{0}$ is set as well. The potential energy will then be modulated only by assigning different $k$ value (i.e., different spring). The 
potential energy of the forces, the states, and the distance between each nucleotide and the imaginary plane were determined in a similar manner as in the cyclization process.

\section{Results}

\subsection{Experiments and Theory}

Figure 3a,b illustrates two, honeycomb and square, lattices in typical DNA origami designs. In a honeycomb lattice, a crossover is placed every $2 / 3$ turn, which corresponds to exactly 7 bp per crossover. This crossover arrangement is compatible with the intrinsic helicity ( $\sim 10.5 \mathrm{bp} /$ turn) of right-handed B-form dsDNA. In contrast, a crossover is placed every $3 / 2$ turns in a square lattice, which equals to $15.75 \mathrm{bp}$ per crossover. The closest integer $16 \mathrm{bp}$ per crossover is typically used, thus yielding $10.67 \mathrm{bp} /$ turn. This will cause a right-handed twist in the global structure. Figure $3 c$ shows the conformation of our DNA origami tile from the FEM simulations with $10.5 \mathrm{bp} /$ turn. A slight, right-handed curvature is observed, thus matching with the lattice design. If the tile is cyclized and the upper and lower edges are sealed with a set of linker staples (shown as the purple line), the structure will have all the dsDNA bundles (shown as gray rods) perfectly aligned as illustrated in Figure 3d.

(a)

(c)
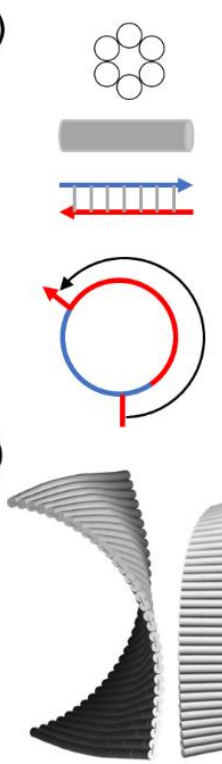

$2 / 3$ turn

$7 \mathrm{bp}$

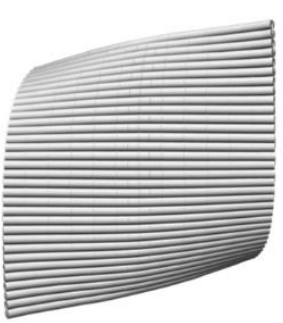

(b)

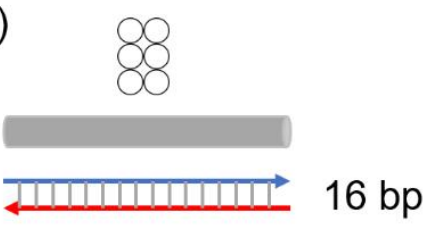

(d)

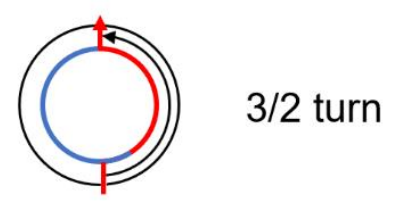

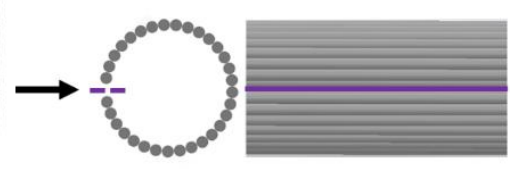

Figure 3. Schematics and FEM simulation results of DNA origami. Comparison of (a) honeycomb and (b) square lattice designs. The gray cylinder represents a dsDNA bundle from the lattice. The blue and red lines with arrows indicate the scaffold and staple strands, respectively, with their $3^{\prime}$ ends marked as arrows. In a honeycomb lattice, crossovers can be placed every $7 \mathrm{bp}$ which is accounted for $2 / 3$ turns in the axial direction of a dsDNA bundle. This corresponds to $10.5 \mathrm{bp} / \mathrm{turn}$, consistent with the helicity of native B-form DNA. In a square lattice, however, a possible crossover is arranged in every $16 \mathrm{bp}$ or $3 / 2$ turns, yielding $10.67 \mathrm{bp} / \mathrm{turn}$. (c) CanDo simulation results of a single-layer DNA origami rectangle in a square lattice (side and front views). With the designed helicity at $10.67 \mathrm{bp} /$ turn, the tile has a minor global twist in the FEM simulations at $10.5 \mathrm{bp} / \mathrm{turn}$. (d) Schematics of the cyclized DNA origami tile in both views. The purple line indicates the sealed upper and lower boundaries by linker staples in experiments.

It is worth noting that the curvature of the DNA origami tile before cyclization cannot be observed under AFM. During deposition, the DNA origami collapses on the mica surface by the attraction force, thus flattening the tile [27]. To see the curvature clearly, multiple tiles may be polymerized side by side into a long ribbon ( $>1 \mu \mathrm{m}$ in the polymerized direction). We previously found that the ribbon also flattens under AFM, but the accumulated twisting leads to a multiple folding of the ribbon and parallelogram-shaped kinks emerge at the 
folded parts [24]. Moreover, the kinks indicate the handedness of the curvature and their density may change if the DNA helicity is altered.

We studied the cyclization of the single-layer DNA origami rectangle designed at $10.67 \mathrm{bp} /$ turn in a series of experiments, as well as theoretical calculations in a previous publication [27]. In the experiments, we added 13 linker staples to the solution containing preformed DNA origami tiles and held at various temperatures $\left(35-50{ }^{\circ} \mathrm{C}\right)$. Then, we measured the origami conformations after different durations of incubation ( 0.5 to $4 \mathrm{~h}$ ). The fractions were determined by counting the origami species based on their conformations (e.g., flat vs. cyclized tiles) in the AFM images. A set of ordinary differential equations (ODEs) was developed to relate the fractions of species as functions of incubation time and temperature. Several kinetic parameters such as reaction rate constants were determined by numerically fitting the experimental data. Along with rate constants, the activation energy of $32.4 \pm 0.7 \mathrm{kcal} / \mathrm{mol}$ was obtained based on the Arrhenius plot [27]. This is the minimum energy needed to overcome barriers and drive the cyclization.

The cyclization process was also studied theoretically [27]. Due to the difficulties in the theoretical calculation of cyclization, the structural transformation was conceptually analyzed as a two-step (flattening and flexing) process. First, the global twist was resolved hypothetically to form a perfectly planar structure by forcing the helicity from 10.5 to $10.67 \mathrm{bp} /$ turn . The twist angle of $8.23^{\circ}$ per $16 \mathrm{bp}$ was calculated considering the difference in the designed and natural DNA helicity (10.67 and $10.5 \mathrm{bp} /$ turn). Given spring constants of dsDNA bundles, the energy needed for flattening the tile was calculated as $\sim 25.6 \mathrm{kcal} / \mathrm{mol}$. Next, the planar structure was bent into an ideally circular shape (as shown in the Figure 3d). This was realized by twisting of dsDNA bundles and bending of crossovers. These two components were modeled as elastic springs connected into a network whose effective spring constant was calculated by considering the springs in parallel and series. With knowledge of the effective spring constant of the entire origami, the energy necessary for bending at $360^{\circ}$ was approximately $6.7 \mathrm{kcal} / \mathrm{mol}$. Combining the two steps, the origami tile transformed from a twist conformation to a planar tile and then to a cyclized tube. Thus, the driving energy for the cyclization process was acquired by summation of the energies for flattening and flexing, yielding $\sim 32.3 \mathrm{kcal} / \mathrm{mol}$ [27]. This theoretical value agrees almost perfectly with the experimentally determined activation energy.

\subsection{Mechanical Energy Induced Cyclization}

Coarse-grained MD simulations were performed to analyze the detailed cyclization process. We realized a series of quasi-equilibrium deformations by defining multiple states and calculating potential energies from one state to the next. First, an equilibrium structure was set as the initial state (state 0 ). Later states were named from 1 to 10 , as shown in Figure 4a. The structure was subject under hypothetical linear spring forces in each process from one state to the next. From state 0 to state 1 , the forces were added between neighboring dsDNA bundles to create the starting conformation of the cyclization (state 1, see Supplementary Material Figure S1). This step was needed since the tile may fold in a random direction toward either side upon external forces, thus the cyclization may not be achieved. For state 1 to 2, 2 to 3, and all the rest until the final state, the forces were added between 13 pairs of cut sites on the linker staples (shown as dots in Figure 1). By pulling the linkers, the upper and lower edges will be brought together, cyclizing the origami tile. 


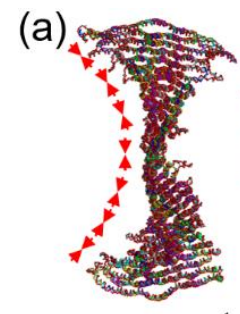

state 0

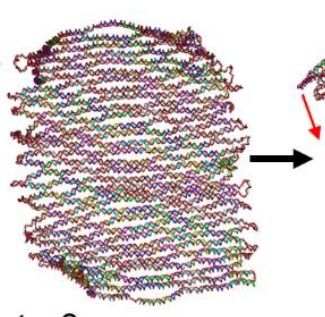

4

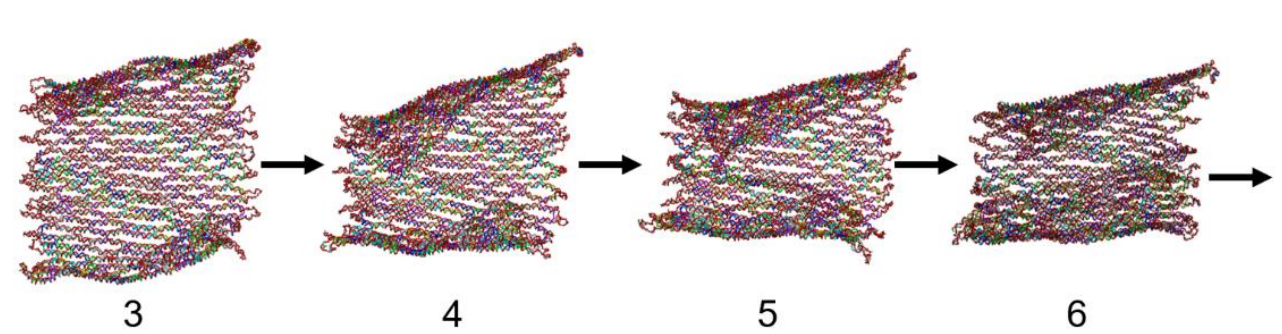

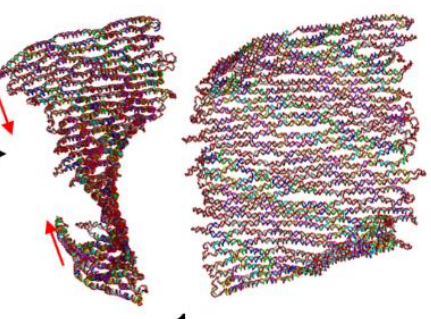

1

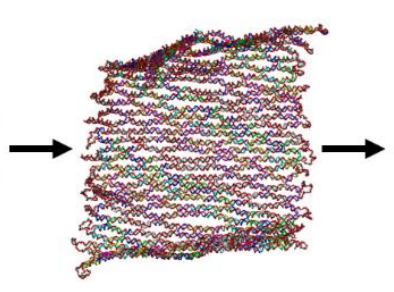

2

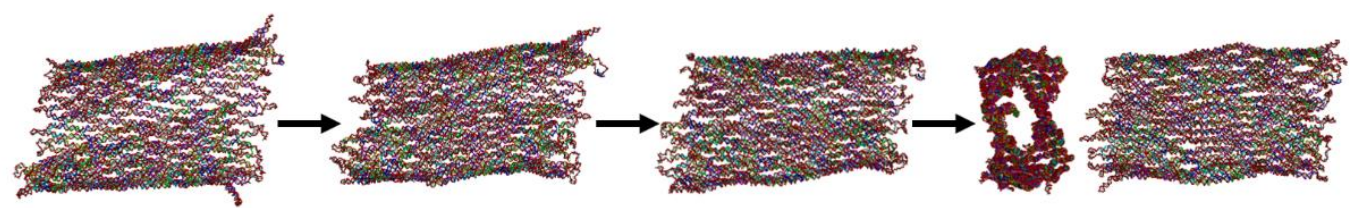

7

8

9

10

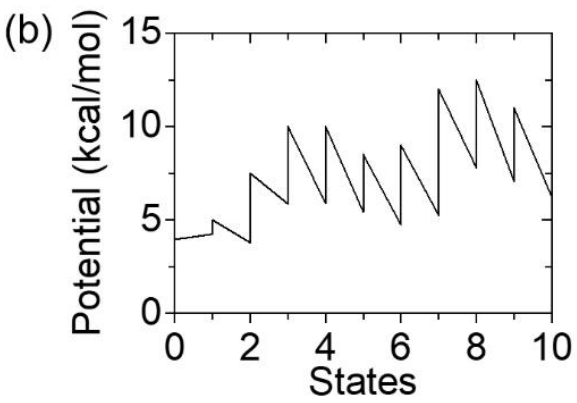

(c)

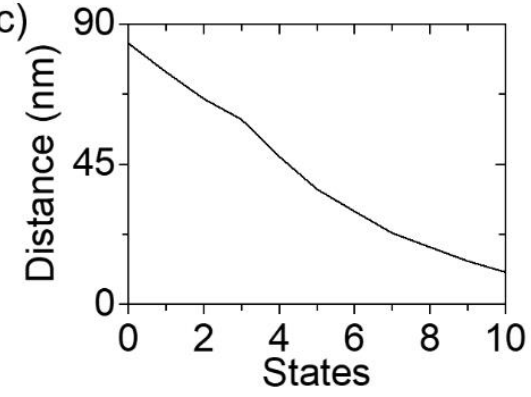

Figure 4. Deformation process of origami tile cyclization in MD simulations. (a) The DNA tile deforms from state 0 (initial) to state 10 (final). In the final state, the tile is cyclized into a tube. The cyclization is induced by adding hypothetical linear spring forces in the MD simulations. From state 0 to state 1 , the forces were added between neighboring dsDNA bundles (illustrated by the red arrows in state 0 ) to create the starting conformation for the cyclization (state 1). From state 1 to 2 , from 2 to 3, and all the rest until the final state, the forces were added between 13 pairs of cut sites on the linker staples (on the upper and lower edges), as illustrated by the red arrows in state 1. (b) Potential energy landscape associated with the applied spring forces for the cyclization. By extracting the initial and final potential energies (i.e., the potential energy of the springs) in a process from one state to the next, the external work is calculated. (c) Distance profile during the cyclization. This is the averaged distance between the cut sites of the linkers. The distance starts from $\sim 84 \mathrm{~nm}$ ( $32 \mathrm{rows}$ of dsDNA bundles or the height of the origami tile) and ends below $11 \mathrm{~nm}$, indicating a cyclized conformation in the final state.

The total number of states were identified from the potential energy calculations. Figure $4 \mathrm{~b}$ shows that each state has two (low and high) potential energies, except for states 0 and 10 (the initial and final states). The low energy is the ending potential energy of the calculation from the previous to the current state, while the high energy is the starting potential energy for the transition from the current state to the next. For example, the potential energies involved in the transition from state 2 to 3 are 7.5 and $5.86 \mathrm{kcal} / \mathrm{mol}$, respectively. That is, the origami in state 2 was subject to a set of external forces with $7.5 \mathrm{kcal} / \mathrm{mol}$, and the minimum potential energy of $5.86 \mathrm{kcal} / \mathrm{mol}$ was found after 2 million calculations. The difference $(1.64 \mathrm{kcal} / \mathrm{mol})$ is the mechanical energy into the DNA origami tile. If the forces were kept constant for further deformation, the upper and lower boundaries would not 
become closer. Therefore, a new stronger set of spring forces was needed (with higher force constant $k$ and lower equilibrium length of the spring $l_{0}$ ). For the transition from state 3 to 4 , multiple potential energies were tried and $10 \mathrm{kcal} / \mathrm{mol}$ was found to further deform the origami without excess energy. Calculations for other states were carried out similarly, by selecting the conformation at the potential minimum in the previous simulation as the starting point of the next computation. The simulations were halted when the upper and lower boundaries were close enough for the linkers to seal them together. Since each linker is $32 \mathrm{nt}$, the base-paired length is estimated as roughly $11 \mathrm{~nm}$. Thus, $11 \mathrm{~nm}$ on average was used as the criterion for the distance between the boundaries. Overall, a total of 10 states were used in the simulations of cyclization.

Under spring forces, the origami tile gradually rolled up and deformed into a cylindrical shape. If a very high potential is given at once (e.g., $400 \mathrm{kcal} / \mathrm{mol}$ ), the tile can cyclize immediately in a single computation (from the initial to the final states). However, such simulations will neither reveal the minimum energy needed for cyclization, nor depict the details of the process. Instead, we used quasi-equilibrium deformations in the simulations. In the early states for cyclization (state 1 through 3), only the dsDNA bundles near the boundaries responded to the pulling forces (Figure 4a). The folding of the entire origami was not strong. In state 4 , all the bundles start to twist and bend. This trend of deformation became more distinct in states 5 through 9 . The global response to the forces suggests that the deformation in the origami propagates gradually. The structure in the final state did not change too much visually compared with that in state 9 . The noticeable change is the alignment of dsDNA bundles in parallel positions. For all states from 1 to 9 , the two boundaries are not parallel to each other, exhibiting a degree of the global twisting. Reaching the final state, they become aligned, indicating that the curvature is fully overcome and the tile cyclizes into a tube as anticipated.

In Figure $4 b$, the lines from one state to the next indicate the energy minimization process, while the vertical lines indicate the increase in the potential energy by introducing new sets of stronger spring forces. All the potential energy changes are added together, and the energy needed for the full cyclization is calculated as $\sim 33.1 \mathrm{kcal} / \mathrm{mol}$. To quantitatively illustrate the cyclization, the averaged distance between the cut site of the linkers on the upper and lower boundaries is plotted in Figure 4c. The distance starts at approximately $84 \mathrm{~nm}$, which is consistent with the designed height $(\sim 88 \mathrm{~nm})$. As the tile cyclizes, the distance reduces steadily and reaches below $11 \mathrm{~nm}$ in the final state. State 10 has $\sim 63 \mathrm{~nm}$ in width and $\sim 25 \mathrm{~nm}$ in diameter, which is comparable to the previous measurement of a cyclized tile $(\sim 65 \times 45 \mathrm{~nm}$ for a flattened tube). [27] This confirms the validity of our approach for cyclization in MD simulations.

\subsection{Energy Driven Planarization}

If an origami tile is designed in a honeycomb lattice (as illustrated in Figure 3a), it will be flat in its intrinsic state. Then, the cyclization process of such a planar origami includes flexing only. For the twisted origami tile (in a square lattice), however, two deformation modes occur simultaneously during the cyclization, overcoming the initial twist and flexing the tile into a tube. It is difficult to separate the two modes. Therefore, we perform MD simulations to study a deformation that purely overcomes the twisting (i.e., flattening). This will also verify the conceptual two-step process in our previous study [27]. All of the 13,360 nucleotides were pulled to the imaginary center flat plane (dashed lines in Figure 5a) by applying the spring forces in a series of quasi-equilibrium deformations. Since the middle part of the origami tile is closer to flat compared with four corners, the imaginary center plane is placed through the middle. Then, strong forces are applied to the middle part in order to hold it in place, while the corners are subject to smaller flattening forces such that they will approach the center plane gradually and steadily. For example, the forces on dsDNA bundle number 12 through 21 are about 3 times stronger than those on the rest of the bundles, when transitioning from state 0 to state 1 . If much stronger forces were applied at the corners, they would be compressed quickly and might be folded instead of moving toward the center plane. In states 
2,3 , and 6, for example, the upper corners hovered on top of the flat part due to thermal fluctuation. They were allowed to deform toward the center plane due to small forces applied instead of folding locally. Moreover, much more energy would have been consumed, thus we could not determine the minimum energy for flattening. Figure 5a shows the gradual deformations of the tile from the twisted curvature to the flat conformation. We observe that the two upper corners along with the top few dsDNA bundles were not in the center plane until the last state. It is worth noting that a step to find the starting conformation was not needed in the flattening, because the origami tile does not have two directions to deform-it deforms only toward the center plane.

(a)
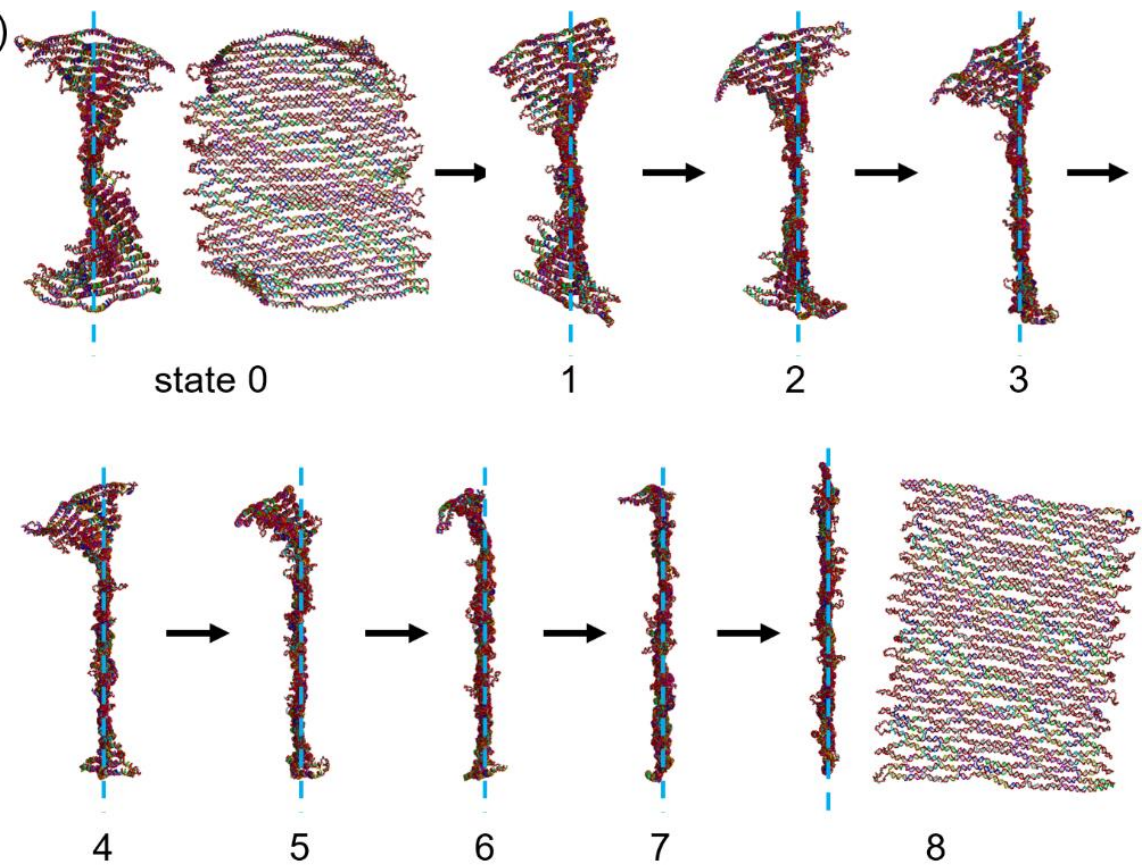

(b)

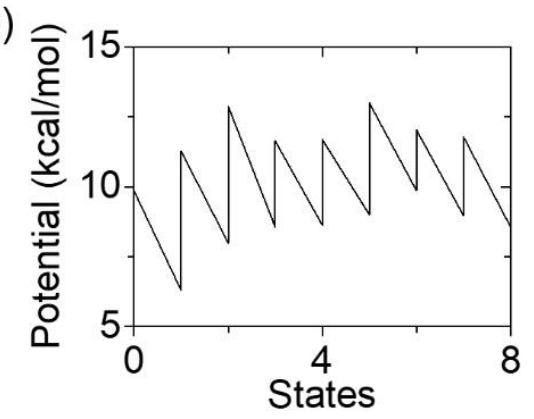

(c)

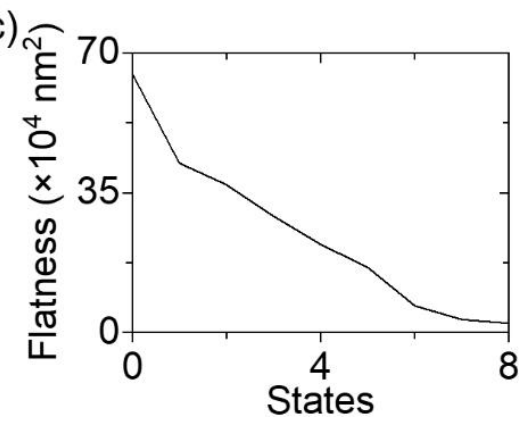

Figure 5. Flattening process of a DNA origami tile in MD simulations. (a) The origami tile flattens from the initial state (state 0) and undergoes gradual planarization until the final state (state 8). The flattening is induced by pulling all the nucleotides to the imaginary flat plane at the center (dashed turquoise line) with hypothetical springs. In the final state, the origami tile is planar. (b) External potential landscape during the flattening process. The external work was calculated in a similar approach as in the cyclization process. (c) Flatness during the flattening. This is a sum of the distance squared of each nucleotide to the imaginary flat plane. Given the radius of a dsDNA bundle $(\sim 1.1 \mathrm{~nm})$, the flatness of a planar tile is $\sim 2 \times 10^{4} \mathrm{~nm}^{2}$. During the deformation, the flatness starts from $\sim 6.5 \times 10^{5} \mathrm{~nm}^{2}$ and ends at $\sim 2.3 \times 10^{4} \mathrm{~nm}^{2}$.

Similar to the cyclization process, the structure was deformed in a multistep process for flattening. The external forces and structural deformation were halted when the origami became flat enough. To develop a criterion, we defined the flatness as the sum of the 
distance squared of each nucleotide to the imaginary flat plane. For example, 0 flatness means perfectly flat. Since the origami tile has a non-zero thickness, its flatness is always greater than 0 . The radius of regular, right-handed B-DNA is $1.1 \mathrm{~nm}$. The flatness is $\sim 2 \times 10^{4} \mathrm{~nm}^{2}$ if we consider a reference state where all the nucleotides are $1.1 \mathrm{~nm}$ from the imaginary center plane. When the flatness of the origami tile is below $2.5 \times 10^{4} \mathrm{~nm}^{2}$, the simulations were halted.

The rectangular origami tile gradually flattened with spring forces toward the plane. As shown in Figure 5a, all parts of the origami response to the forces in all the states. For all states from 0 to 8 , the middle part stays in the center plane while all the corners gradually approach the plane. The energy is calculated in the same method as for the cyclization in Figure 4. The potential energy landscape and the flatness suggest that about $26.3 \mathrm{kcal} / \mathrm{mol}$ is required to flatten the origami tile (Figure $5 b, c)$. During the flattening, the flatness starts from $\sim 6.5 \times 10^{5} \mathrm{~nm}^{2}$ and ends at $\sim 2.3 \times 10^{4} \mathrm{~nm}^{2}$ (within the criterion) The conformation in the final state measures around $86 \mathrm{~nm}$ in height and $63 \mathrm{~nm}$ in width, showing good agreement with our design and AFM imaging results [27].

\section{Discussion}

\subsection{Equilibrium Conformations in CanDo and OxDNA}

The results in Figure $3 c$ and the initial state (state 0 ) in Figure 4 a are slightly different. The dsDNA bundles are closely packed in the structure from CanDo, whereas gaps are seen in the oxDNA results. Thus, the origami height in Figure $3 \mathrm{c}$ appears to be shorter than that in Figure $4 \mathrm{a}$. Several factors are accounted for these differences. FEM simulations in CanDo have the single base-pair resolution, treating ssDNA and crossovers as a connection without any length. These make the DNA origami stiffer than it is. For example, the crossovers are not seen in Figure $3 c$ since they have no length. Similarly, the unpaired scaffold domains at either side of the tile are not shown as fluffy segments. In contrast, oxDNA adopts the coarse-grained MD model, where each nucleotide is the basic group of atoms (i.e., single nucleotide resolution). The MD model accounts for several parameters in the simulations, including temperature, salt concentration, and major or minor groove of dsDNA. OxDNA provides significantly more detailed information than CanDo. For example, the neighboring dsDNA bundles connected by crossovers will stay together. However, the segments of neighboring bundles with no crossovers will show gaps in oxDNA, indicating the repulsion between dsDNA helices due to negative charges. This is not depicted in CanDo. Because of the gaps, the height in oxDNA is greater than that of CanDo, causing the difference in size. Aside from these differences, both conformations are comparable.

\subsection{Configuration of a Cyclized Origami Tile}

The final configuration of the origami tile in Figure 4 a slightly differs from that in Figure $3 \mathrm{~d}$. There are several reasons for the difference. The schematics in Figure $3 \mathrm{~d}$ show the ideal configuration of the cyclized structure, where all the dsDNA bundles are perfectly aligned. In the oxDNA, however, the dsDNA bundles are bent slightly, the unpaired ssscaffold segments cause attraction or repulsion forces, and the thermal fluctuation changes the overall conformation to some degree. The differences are limited, nevertheless. The cross-section of the MD simulation results resembles a circular tube as in Figure $3 \mathrm{~d}$. The upper and lower surface of the tube are parallel to each other. Finally, the size of the tube (about $63 \mathrm{~nm}$ in width and $25 \mathrm{~nm}$ in diameter) also agrees with the previous experimental results [27].

\subsection{Deformation Process of Cyclization}

Figure $4 \mathrm{a}$ shows one possible deformation pathway of cyclization with minimal energy. Overcoming of the twisting and bending of the structure occur simultaneously. The simulations also reveal the gradual propagation of the deformation in the structure during the cyclization. This information is available only with computational studies, whereas ex- 
periments show the origami conformations before and after cyclization. In addition, the theoretical approach cannot consider the cyclization process directly, rather it introduces a hypothetical intermediate step. Thus, MD simulations can provide detailed information such as the conformation and necessary energy during the cyclization process as well as pathways.

\subsection{Free Energy Change in Cyclization}

The cyclization process was studied experimentally and theoretically in our previous report [27], as well as by MD simulations in this work. As discussed above, the cyclization was theorized into two independent processes of flattening and flexing by introducing a hypothetical intermediate planar conformation. The energy for cyclization equals the summation of energy changes for flattening and flexing:

$$
E_{\text {cyclization }}=E_{\text {flattening }}+E_{\text {flexing }}
$$

Figure 6 summarizes the cyclization and the conceptual two-step process. The energies extracted from all three approaches are in remarkable agreement. Note that it was impossible to determine the energies needed for flattening or flexing in experiments. The mechanical analysis and MD computation yielded $E_{\text {flattening }}$ of approximately 25.6 and $26.3 \mathrm{kcal} / \mathrm{mol}$, respectively. It is worth noting that flexing was not performed in MD simulations, because the origami could twist again once subjected under flexing forces. For any given initial and final states, various deformation pathways may be proposed to calculate the related energies. However, whether the process is realistic or not may not be revealed by the theory alone, even if it is a free energy driven reaction. Therefore, performing simulations along with the theory will be beneficial.
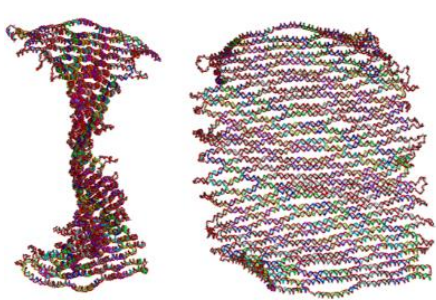

\section{Cyclization}
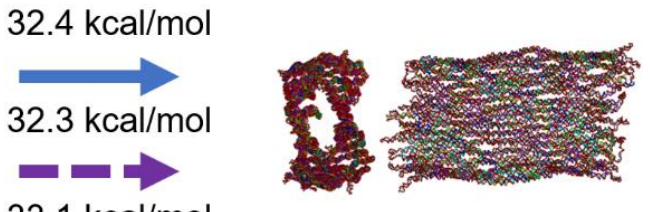

$33.1 \mathrm{kcal} / \mathrm{mol}$

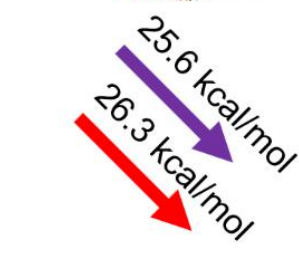

Flattening

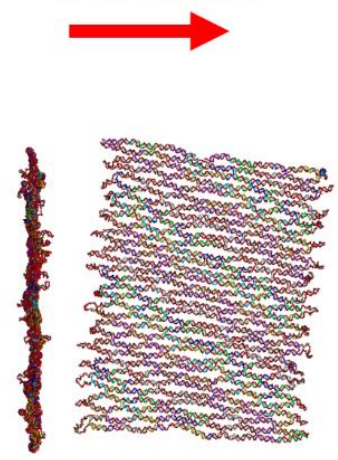

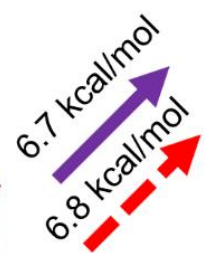

Flexing

Figure 6. Overall analysis of the cyclization process. The cyclization of a DNA origami tile with a twist may be conceptualized as a two-step (flattening and flexing) process by introducing a hypothetical intermediate planar conformation. Blue, purple, and red arrows indicate the experiment, theory, and simulation, respectively. Solid arrows denote the direct results, while dashed arrows mark the indirect results from summation or subtraction of direct results. Experimentally determined free energy change for cyclization is approximately $32.4 \mathrm{kcal} / \mathrm{mol}$. The theoretical value for the cyclization energy is obtained as $\sim 32.3 \mathrm{kcal} / \mathrm{mol}$ by adding energies for flattening and flexing steps. The MD simulations yield a cyclization energy of about $33.1 \mathrm{kcal} / \mathrm{mol}$, consistent with the experimental and theoretical values.

Since direct numerical studies on flexing are not practical, the flexing energy may be obtained from Equation 7 using the cyclization and flattening energies, which is about 
$6.8 \mathrm{kcal} / \mathrm{mol}$. This value also agrees well with the theoretical value of $\sim 6.7 \mathrm{kcal} / \mathrm{mol}$. The combined experiment, theory, and computation all agree well and verify the effectiveness of the simulation approach in this work.

In summary, coarse-grained MD simulations were performed on the oxDNA platform to provide critical insights on the cyclization of a DNA origami tile with initial twisting. This work advances our understanding of fundamental mechanics of DNA origami in general and should be useful for energy driven processes including adaptive reconfiguration, mechanical (or other) energy absorption mechanisms, and the interactions with other biomolecules. For example, the energy consumption determined by numerical simulations may be used to design the length of linkers and the $C-G$ ratio for reconfiguration of an origami structure. Mobile nanodevices such as DNA walkers could also be designed and optimized by the computation of energy landscape [59-62].

Supplementary Materials: The following are available online at https:/ / www.mdpi.com/2076-3 417/11/5/2357/s1, Figure S1: Starting conformation for cyclization, Figure S2: Potential energy landscape for the cyclization of the DNA origami tile, Figure S3: Potential energy landscape for the flattening of the DNA origami tile.

Author Contributions: Conceptualization, R.L., H.C. and J.H.C.; methodology, R.L.; software, R.L.; validation, R.L. and H.L.; formal analysis, R.L.; investigation, R.L.; resources, R.L.; data curation, R.L.; writing - original draft preparation, R.L.; writing — review and editing, R.L. and J.H.C.; visualization, R.L.; supervision, J.H.C.; project administration, J.H.C.; funding acquisition, J.H.C. All authors have read and agreed to the published version of the manuscript.

Funding: This work was funded by the U.S. Department of Energy (DOE), Office of Science, Basic Energy Sciences (BES), under award no. DE-SC0020673 (computational studies) and by the U.S. National Science Foundation (NSF) under award no. 1710344 (experimental analysis).

Institutional Review Board Statement: Not applicable.

Informed Consent Statement: Not applicable.

Data Availability Statement: The data presented in this study are available in Supplementary Materials.

Conflicts of Interest: The authors declare no conflict of interest.

\section{References}

1. Seeman, N.C.; Sleiman, H.F. DNA Nanotechnology. Nat. Rev. Mater. 2017, 3, 1-23.

2. Pinheiro, A.V.; Han, D.; Shih, W.M.; Yan, H. Challenges and Opportunities for Structural DNA Nanotechnology. Nat. Nanotechnol. 2011, 6, 763-772. [CrossRef] [PubMed]

3. Seeman, N.C. Nucleic Acid Junctions and Lattices. J. Theor. Biol. 1982, 99, 237-247. [CrossRef]

4. Lin, C.; Liu, Y.; Rinker, S.; Yan, H. DNA Tile Based Self-Assembly: Building Complex Nanoarchitectures. ChemPhysChem 2006, 7, 1641-1647. [CrossRef]

5. Park, S.H.; Pistol, C.; Ahn, S.J.; Reif, J.H.; Lebeck, A.R.; Dwyer, C.; LaBean, T.H. Finite-Size, Fully Addressable DNA Tile Lattices Formed by Hierarchical Assembly Procedures. Angew. Chem. 2006, 118, 749-753. [CrossRef]

6. Wei, B.; Dai, M.; Yin, P. Complex Shapes Self-Assembled from Single-Stranded DNA Tiles. Nature 2012, 485, 623-626. [CrossRef] [PubMed]

7. Ke, Y.; Ong, L.L.; Shih, W.M.; Yin, P. Three-Dimensional Structures Self-Assembled from DNA Bricks. Science 2012, 338, 1177-1183. [CrossRef] [PubMed]

8. Ke, Y.; Ong, L.L.; Sun, W.; Song, J.; Dong, M.; Shih, W.M.; Yin, P. DNA Brick Crystals with Prescribed Depths. Nat. Chem. 2014, 6, 994-1002. [CrossRef] [PubMed]

9. Yurke, B.; Turberfield, A.J.; Mills, A.P.; Simmel, F.C.; Neumann, J.L. A DNA-Fuelled Molecular Machine Made of DNA. Nature 2000, 406, 605-608. [CrossRef]

10. Liu, M.; Fu, J.; Hejesen, C.; Yang, Y.; Woodbury, N.W.; Gothelf, K.; Liu, Y.; Yan, H. A DNA Tweezer-Actuated Enzyme Nanoreactor. Nat. Commun. 2013, 4, 1-5. [CrossRef]

11. Omabegho, T.; Sha, R.; Seeman, N.C. A Bipedal DNA Brownian Motor with Coordinated Legs. Science 2009, 324, 67-71. [CrossRef]

12. Lund, K.; Manzo, A.J.; Dabby, N.; Michelotti, N.; Johnson-Buck, A.; Nangreave, J.; Taylor, S.; Pei, R.; Stojanovic, M.N.; Walter, N.G. Molecular Robots Guided by Prescriptive Landscapes. Nature 2010, 465, 206-210. [CrossRef]

13. Pan, J.; Cha, T.-G.; Li, F.; Chen, H.; Bragg, N.A.; Choi, J.H. Visible/Near-Infrared Subdiffraction Imaging Reveals the Stochastic Nature of DNA Walkers. Sci. Adv. 2017, 3, e1601600. [CrossRef] [PubMed] 
14. Pan, J.; Du, Y.; Qiu, H.; Upton, L.R.; Li, F.; Choi, J.H. Mimicking Chemotactic Cell Migration with DNA Programmable Synthetic Vesicles. Nano Lett. 2019, 19, 9138-9144. [CrossRef] [PubMed]

15. Zhang, D.Y.; Seelig, G. Dynamic DNA Nanotechnology Using Strand-Displacement Reactions. Nat. Chem. 2011, 3, 103-113. [CrossRef] [PubMed]

16. Li, F.; Chen, H.; Pan, J.; Cha, T.-G.; Medintz, I.L.; Choi, J.H. A DNAzyme-Mediated Logic Gate for Programming Molecular Capture and Release on DNA Origami. Chem. Commun. 2016, 52, 8369-8372. [CrossRef]

17. Zhang, D.Y.; Winfree, E. Control of DNA Strand Displacement Kinetics Using Toehold Exchange. J. Am. Chem. Soc. 2009, 131, 17303-17314. [CrossRef]

18. Rothemund, P.W.K. Folding DNA to Create Nanoscale Shapes and Patterns. Nature 2006, 440, 297-302. [CrossRef]

19. Grima, J.N.; Mizzi, L.; Azzopardi, K.M.; Gatt, R. Auxetic Perforated Mechanical Metamaterials with Randomly Oriented Cuts. Adv. Mater. 2016, 28, 385-389. [CrossRef]

20. Castro, C.E.; Kilchherr, F.; Kim, D.-N.; Shiao, E.L.; Wauer, T.; Wortmann, P.; Bathe, M.; Dietz, H. A Primer to Scaffolded DNA Origami. Nat. Methods 2011, 8, 221. [CrossRef]

21. Kopperger, E.; List, J.; Madhira, S.; Rothfischer, F.; Lamb, D.C.; Simmel, F.C. A Self-Assembled Nanoscale Robotic Arm Controlled by Electric Fields. Science 2018, 359, 296-301. [CrossRef]

22. Choi, J.; Chen, H.; Li, F.; Yang, L.; Kim, S.S.; Naik, R.R.; Ye, P.D.; Choi, J.H. Nanomanufacturing of 2D Transition Metal Dichalcogenide Materials Using Self-Assembled DNA Nanotubes. Small 2015, 11, 5520-5527. [CrossRef]

23. Li, R.; Chen, H.; Choi, J.H. Topological Assembly of a Deployable Hoberman Flight Ring from DNA. Small 2021, 17, 2007069. [CrossRef]

24. Chen, H.; Zhang, H.; Pan, J.; Cha, T.-G.; Li, S.; Andréasson, J.; Choi, J.H. Dynamic and Progressive Control of DNA Origami Conformation by Modulating DNA Helicity with Chemical Adducts. ACS Nano 2016, 10, 4989-4996. [CrossRef]

25. Czogalla, A.; Petrov, E.P.; Kauert, D.J.; Uzunova, V.; Zhang, Y.; Seidel, R.; Schwille, P. Switchable Domain Partitioning and Diffusion of DNA Origami Rods on Membranes. Faraday Discuss. 2013, 161, 31-43. [CrossRef]

26. Mishra, S.; Feng, Y.; Endo, M.; Sugiyama, H. Advances in DNA Origami-Cell Interfaces. ChemBioChem 2020, 21, 33-44. [CrossRef] [PubMed]

27. Chen, H.; Weng, T.-W.; Riccitelli, M.M.; Cui, Y.; Irudayaraj, J.; Choi, J.H. Understanding the Mechanical Properties of DNA Origami Tiles and Controlling the Kinetics of Their Folding and Unfolding Reconfiguration. J. Am. Chem. Soc. 2014, 136, 6995-7005. [CrossRef]

28. Li, Z.; Liu, M.; Wang, L.; Nangreave, J.; Yan, H.; Liu, Y. Molecular Behavior of DNA Origami in Higher-Order Self-Assembly. J. Am. Chem. Soc. 2010, 132, 13545-13552. [CrossRef]

29. Liber, M.; Tomov, T.E.; Tsukanov, R.; Berger, Y.; Nir, E. A Bipedal DNA Motor that Travels Back and Forth Between Two DNA Origami Tiles. Small 2015, 11, 568-575. [CrossRef] [PubMed]

30. Zhao, Z.; Yan, H.; Liu, Y. A Route to Scale up DNA Origami Using DNA Tiles as Folding Staples. Angew. Chem. 2010, 122, 1456-1459. [CrossRef]

31. Andersen, E.S.; Dong, M.; Nielsen, M.M.; Jahn, K.; Subramani, R.; Mamdouh, W.; Golas, M.M.; Sander, B.; Stark, H.; Oliveira, C.L. Self-Assembly of a Nanoscale DNA Box with a Controllable Lid. Nature 2009, 459, 73-76. [CrossRef]

32. Zadegan, R.M.; Jepsen, M.D.; Thomsen, K.E.; Okholm, A.H.; Schaffert, D.H.; Andersen, E.S.; Birkedal, V.; Kjems, J. Construction of a 4 Zeptoliters Switchable 3D DNA Box Origami. ACS Nano 2012, 6, 10050-10053. [CrossRef] [PubMed]

33. Kuzuya, A.; Komiyama, M. Design and Construction of a Box-Shaped 3D-DNA Origami. Chem. Commun. 2009, $28,4182-4184$. [CrossRef] [PubMed]

34. Zhang, F.; Jiang, S.; Wu, S.; Li, Y.; Mao, C.; Liu, Y.; Yan, H. Complex Wireframe DNA Origami Nanostructures with Multi-Arm Junction Vertices. Nat. Nanotechnol. 2015, 10, 779-784. [CrossRef]

35. Benson, E.; Mohammed, A.; Rayneau-Kirkhope, D.; Gådin, A.; Orponen, P.; Högberg, B. Effects of Design Choices on the Stiffness of Wireframe DNA Origami Structures. ACS Nano 2018, 12, 9291-9299. [CrossRef]

36. Veneziano, R.; Ratanalert, S.; Zhang, K.; Zhang, F.; Yan, H.; Chiu, W.; Bathe, M. Designer Nanoscale DNA Assemblies Programmed from the Top Down. Science 2016, 352, 1534. [CrossRef]

37. Ke, Y.; Castro, C.; Choi, J.H. Structural DNA Nanotechnology: Artificial Nanostructures for Biomedical Research. Ann. Rev. Biomed. Eng. 2018, 20, 375-401. [CrossRef]

38. Ijäs, H.; Nummelin, S.; Shen, B.; Kostiainen, M.A.; Linko, V. Dynamic DNA Origami Devices: From Strand-Displacement Reactions to External-Stimuli Responsive Systems. Int. J. Mol. Sci. 2018, 19, 2114. [CrossRef] [PubMed]

39. Zhang, Y.; Pan, V.; Li, X.; Yang, X.; Li, H.; Wang, P.; Ke, Y. Dynamic DNA Structures. Small 2019, 15, 1900228. [CrossRef]

40. Chen, H.; Cha, T.-G.; Pan, J.; Choi, J.H. Hierarchically Assembled DNA Origami Tubules with Reconfigurable Chirality. Nanotechnology 2013, 24, 435601. [CrossRef] [PubMed]

41. Zhang, Z.; Yang, Y.; Pincet, F.; Llaguno, M.C.; Lin, C. Placing and Shaping Liposomes with Reconfigurable DNA Nanocages. Nat. Chem. 2017, 9, 653-659. [CrossRef] [PubMed]

42. Zhang, F.; Nangreave, J.; Liu, Y.; Yan, H. Reconfigurable DNA Origami to Generate Quasifractal Patterns. Nano Lett. 2012, 12, 3290-3295. [CrossRef]

43. Kauert, D.J.; Kurth, T.; Liedl, T.; Seidel, R. Direct Mechanical Measurements Reveal the Material Properties of Three-Dimensional DNA Origami. Nano Lett. 2011, 11, 5558-5563. [CrossRef] [PubMed] 
44. Oberstrass, F.C.; Fernandes, L.E.; Bryant, Z. Torque Measurements Reveal Sequence-Specific Cooperative Transitions in Supercoiled DNA. Proc. Natl. Acad. Sci. USA 2012, 109, 6106-6111. [CrossRef] [PubMed]

45. Sa-Ardyen, P.; Vologodskii, A.V.; Seeman, N.C. The Flexibility of DNA Double Crossover Molecules. Biophys. J. 2003, 84, 3829-3837. [CrossRef]

46. Li, R.; Chen, H.; Choi, J.H. Auxetic Two-Dimensional Nanostructures from DNA. Angew. Chem. 2021. [CrossRef]

47. Goodman, R.P.; Schaap, I.A.; Tardin, C.F.; Erben, C.M.; Berry, R.M.; Schmidt, C.F.; Turberfield, A.J. Rapid Chiral Assembly of Rigid DNA Building Blocks for Molecular Nanofabrication. Science 2005, 310, 1661-1665. [CrossRef] [PubMed]

48. Douglas, S.M.; Marblestone, A.H.; Teerapittayanon, S.; Vazquez, A.; Church, G.M.; Shih, W.M. Rapid Prototyping of 3D DNA-Origami Shapes with CaDNAno. Nucleic Acids Res. 2009, 37, 5001-5006. [CrossRef]

49. Dietz, H.; Douglas, S.M.; Shih, W.M. Folding DNA into Twisted and Curved Nanoscale Shapes. Science 2009, 325, 725-730. [CrossRef]

50. Wagenbauer, K.F.; Sigl, C.; Dietz, H. Gigadalton-Scale Shape-Programmable DNA Assemblies. Nature 2017, 552, 78-83. [CrossRef]

51. Chen, H.; Li, R.; Li, S.; Andréasson, J.; Choi, J.H. Conformational Effects of UV Light on DNA Origami. J. Am. Chem. Soc. 2017, 139, 1380-1383. [CrossRef] [PubMed]

52. Ke, Y.; Bellot, G.; Voigt, N.V.; Fradkov, E.; Shih, W.M. Two Design Strategies for Enhancement of Multilayer-DNA-Origami Folding: Underwinding for Specific Intercalator Rescue and Staple-Break Positioning. Chem. Sci. 2012, 3, 2587-2597. [CrossRef] [PubMed]

53. Zadegan, R.M.; Lindau, E.G.; Klein, W.P.; Green, C.; Graugnard, E.; Yurke, B.; Kuang, W.; Hughes, W.L. Twisting of DNA Origami from Intercalators. Sci. Rep. 2017, 7, 1-5. [CrossRef] [PubMed]

54. Snodin, B.E.K.; Randisi, F.; Mosayebi, M.; Šulc, P.; Schreck, J.S.; Romano, F.; Ouldridge, T.E.; Tsukanov, R.; Nir, E.; Louis, A.A.; et al. Introducing Improved Structural Properties and Salt Dependence into a Coarse-Grained Model of DNA. J. Chem. Phys. 2015, 142, 234901. [CrossRef] [PubMed]

55. Engel, M.C.; Smith, D.M.; Jobst, M.A.; Sajfutdinow, M.; Liedl, T.; Romano, F.; Rovigatti, L.; Louis, A.A.; Doye, J.P.K. Force-Induced Unravelling of DNA Origami. ACS Nano 2018, 12, 6734-6747. [CrossRef] [PubMed]

56. Snodin, B.E.; Schreck, J.S.; Romano, F.; Louis, A.A.; Doye, J.P. Coarse-Grained Modelling of the Structural Properties of DNA Origami. Nucleic Acids Res. 2019, 47, 1585-1597. [CrossRef]

57. Snodin, B.E.; Romano, F.; Rovigatti, L.; Ouldridge, T.E.; Louis, A.A.; Doye, J.P. Direct Simulation of the Self-Assembly of a Small DNA Origami. ACS Nano 2016, 10, 1724-1737. [CrossRef]

58. Shi, Z.; Castro, C.E.; Arya, G. Conformational Dynamics of Mechanically Compliant DNA Nanostructures from Coarse-Grained Molecular Dynamics Simulations. ACS Nano 2017, 11, 4617-4630. [CrossRef]

59. Cha, T.-G.; Pan, J.; Chen, H.; Salgado, J.; Li, X.; Mao, C.; Choi, J.H. A Synthetic DNA Motor that Transports Nanoparticles along Carbon Nanotubes. Nat. Nanotechnol. 2014, 9, 39-43. [CrossRef]

60. Cha, T.-G.; Pan, J.; Chen, H.; Robinson, H.N.; Li, X.; Mao, C.; Choi, J.H. Design Principles of DNA Enzyme-Based Walkers: Translocation Kinetics and Photoregulation. J. Am. Chem. Soc. 2015, 137, 9429-9437. [CrossRef]

61. Li, F.; Cha, T.-G.; Pan, J.; Ozcelikkale, A.; Han, B.; Choi, J.H. DNA Walker-Regulated Cancer Cell Growth Inhibition. ChemBioChem 2016, 17, 1138-1141. [CrossRef] [PubMed]

62. Du, Y.; Pan, J.; Qiu, H.; Mao, C.; Choi, J.H. Mechanistic Understanding of Surface Migration Dynamics with DNA Walkers. J. Phys. Chem. B 2021, 125, 507-517. [CrossRef] [PubMed] 